\title{
Axial asymmetry in the IVBM
}

\author{
H. G. Ganev ${ }^{1}$ a \\ Joint Institute for Nuclear Research, 141980 Dubna, Moscow Region, Russia
}

Received: date / Revised version: date

\begin{abstract}
The dynamical symmetry limit of the two-fluid Interacting Vector Boson Model (IVBM), defined through the chain $S p(12, R) \supset U(3,3) \supset U_{p}(3) \otimes \overline{U_{n}(3)} \supset S U^{*}(3) \supset S O(3)$, is considered and applied for the description of nuclear collective spectra exhibiting axially asymmetric features. The effect of the introduction of a Majorana interaction to the $S U^{*}(3)$ model Hamiltonian on the $\gamma$-band energies is studied. The theoretical predictions are compared with the experimental data for ${ }^{192} \mathrm{Os},{ }^{190} \mathrm{Os}$, and ${ }^{112} \mathrm{Ru}$ isotopes. It is shown that by taking into account the full symplectic structures in the considered dynamical symmetry of the IVBM, the proper description of the energy spectra and the $\gamma$-band energy staggering of the nuclei under considerations can be achieved. The obtained results show that the potential energy surfaces for the following two nuclei ${ }^{192} \mathrm{Os}$ and ${ }^{112} \mathrm{Ru}$, possess almost $\gamma$-flat potentials with very shallow triaxial minima, suggesting a more complex and intermediate situation between $\gamma$-rigid and $\gamma$-unstable structures. Additionally, the absolute $B(E 2)$ intraband transition probabilities between the states of the ground state band and $\gamma$ band, as well as the $B(M 1)$ interband transition probabilities between the states of the ground and $\gamma$ bands for the two nuclei ${ }^{192} \mathrm{Os}$ and ${ }^{190} \mathrm{Os}$ are calculated and compared with experiment and for the $B(E 2)$ values with the predictions of some other collective models incorporating the $\gamma$-rigid or $\gamma$-unstable structures. The obtained results agree well with the experimental data and reveal the relevance of the used dynamical symmetry of IVBM in the description of nuclei exhibiting axially asymmetric features in their spectra.
\end{abstract}

PACS. 21.60.Fw Models based on group theory - 21.10.Re Collective levels

\section{Introduction}

It has been known for a long time that in certain mass regions nuclei with static deformation show deviations from a rigid axially symmetric picture. The possibility of static triaxial shapes for the ground state of nuclei is a longstanding problem in nuclear structure physics despite the fact that very few candidates have been found experimentally [1, 2]. In the geometrical approach the triaxial nuclear properties are usually interpreted in terms of either the $\gamma$-unstable rotor model of Wilets and Jean 3 or the rigid triaxial rotor model (RTRM) of Davydov et al. [4. These models exploit the geometrical picture of nucleus according to the Collective Model of Bohr and Mottelson, expressed in terms of the intrinsic variables $\beta$ and $\gamma$ where the former specifies the ellipsoidal quadrupole deformation and the latter the degree of axial asymmetry. To describe the deviations from axial symmetry the model of Wilets and Jean assumes that the potential energy is independent of the $\gamma$-degree of freedom, while in the model of Davydov et al. one considers a harmonic oscillator potential with a

${ }^{a}$ On leave of absence from the Institute of Nuclear Research and Nuclear Energy, Bulgarian Academy of Sciences, Sofia, Bulgaria minimum at finite values of $\gamma$ producing a rigid triaxial shape of the nucleus.

The question of whether asymmetric atomic nuclei are $\gamma$-unstable or $\gamma$-rigid has been an ongoing and active issue in nuclear structure physics for over half a century. A number of signatures of $\gamma$-unstable and $\gamma$-rigid structures in nuclei has been discussed [1, [2], [5]-[8]. While it might be thought that the potential energy surfaces that are nearly $\gamma$-flat or which display deep minima for some value of $\gamma$ would produce rather different nuclear spectra, this is in fact not the case. Indeed, the predictions for $\gamma$-unstable and $\gamma$-rigid potentials are nearly identical for most observables if the average value of $\gamma$ in the first case, $\gamma_{r m s}$, is equal to the $\gamma_{\text {rigid }}$ in the second, a situation occurring for example in the Os-Pt region. However, a clear distinction arises in the $\gamma$ band, where both the $\gamma$-unstable and $\gamma$-rigid models exhibit an opposite energy staggering. The comparison of a $\gamma$-rigid rotor and a $\gamma$-unstable models yields similar ground state band energies, but the levels of $\gamma$-band are grouped as $2^{+},\left(3^{+}, 4^{+}\right),\left(5^{+}, 6^{+}\right), \ldots$ in $\gamma$ unstable and as $\left(2^{+}, 3^{+}\right),\left(4^{+}, 5^{+}\right), \ldots$ in $\gamma$-rigid models, respectively. Thus, obviously the structure of the $\gamma$ band is crucial for the identification of the shape in the real nuclei and hence for the manifestation of the $\gamma$ degrees of freedom. 
In Ref. 9] a new dynamical symmetry limit of the twofluid Interacting Vector Boson Model (IVBM) was introduced, which seems to be appropriate for the description of deformed even-even nuclei, exhibiting triaxial features. It was shown there that the addition of Majorana interaction to the $S U^{*}(3)$ model Hamiltonian produces a stable triaxial minimum. In this paper, we develop further our theoretical approach initiated in Ref. 9 by considering in more details the spectra of some even-even transitional nuclei, supposed to be axially asymmetric, in the framework of the symplectic IVBM with $S p(12, R)$ as a group of dynamical symmetry. We focus on the $\gamma$-band properties and show how the $\gamma$-band energies (and the corresponding energy staggering) are affected by the presence of the introduced interaction. The theoretical predictions are compared with the experimental data for ${ }^{192} \mathrm{Os},{ }^{190} \mathrm{Os}$, and ${ }^{112} R u$ isotopes. It will be shown that by taking into account the full symplectic structures in the considered dynamical symmetry of the IVBM, the proper description of the energy spectra and the $\gamma$-band energy staggering of the nuclei under considerations can be achieved.

Additionally, the absolute $B(E 2)$ intraband transition probabilities between the states of the ground state band and $\gamma$ band, as well as the $B(M 1)$ interband transition probabilities between the states of the ground and $\gamma$ bands for the two nuclei ${ }^{192} \mathrm{Os}$ and ${ }^{190} \mathrm{Os}$ are considered and compared with experiment and for the $B(E 2)$ values with the predictions of some other collective models incorporating the $\gamma$-rigid or $\gamma$-unstable structures.

\section{The algebraic structure of the $U(3,3)$ dynamical symmetry}

It was suggested by Bargmann and Moshinsky [10, [11 that two types of bosons are needed for the description of nuclear dynamics. It was shown there that the consideration of only two-body system consisting of two different interacting vector particles will suffice to give a complete description of $N$ three-dimensional oscillators with a quadrupole-quadrupole interaction. The latter can be considered as the underlying basis in the algebraic construction of the phenomenological IVBM.

The algebraic structure of the IVBM [12] is realized in terms of creation and annihilation operators of two kinds of vector bosons $u_{m}^{\dagger}(\alpha), u_{m}(\alpha)(m=0, \pm 1)$, which differ in an additional quantum number $\alpha= \pm 1 / 2$ (or $\alpha=p$ and $n$ )-the projection of the $T$-spin (an analogue to the $F$-spin of IBM-2 or the $I$-spin of the particle-hole IBM). In the present paper, we consider these two bosons just as elementary building blocks or quanta of elementary excitations (phonons) rather than real fermion pairs, which generate a given type of algebraic structures. Thus, only their tensorial structure is of importance and they are used as an auxiliary tool, generating an appropriate dynamical symmetry.

We consider the following reduction chain of the dynamical symmetry group $S p(12, R)$ of the IVBM for study- ing the triaxiality in atomic nuclei:

$$
\begin{array}{ccccc}
S p(12, R) & \supset & U(3,3) & & \\
& & & \\
& \supset & U_{p}(3) & \otimes & \overline{U_{n}(3)} \\
& {\left[N_{p}\right]_{3}} & & {\left[-N_{n}\right]_{3}} \\
& & & \\
& & S U^{*}(3) & \supset & S O(3), \\
& (\lambda, \mu) & K & L
\end{array}
$$

where the labels below the different subgroups are the quantum numbers corresponding to their irreducible representations (irreps). As it was shown in Ref. 9], this dynamical symmetry is appropriate for nuclei in which the one type of particles is particle-like and the other is holelike. For more details concerning the algebraic structure of this dynamical symmetry of the IVBM, we refer the readers to Ref. 9 .

All bilinear combinations of the creation and annihilation operators of the two vector bosons generate the boson representations of the non-compact symplectic group $S p(12, R)$ :

$$
\begin{aligned}
& F_{M}^{L}(\alpha, \beta)=\sum_{k, m} C_{1 k 1 m}^{L M} u_{k}^{+}(\alpha) u_{m}^{+}(\beta), \\
& G_{M}^{L}(\alpha, \beta)=\sum_{k, m} C_{1 k 1 m}^{L M} u_{k}(\alpha) u_{m}(\beta), \\
& A_{M}^{L}(\alpha, \beta)=\sum_{k, m} C_{1 k 1 m}^{L M} u_{k}^{+}(\alpha) u_{m}(\beta),
\end{aligned}
$$

where $C_{1 k 1 m}^{L M}$, which are the usual Clebsch-Gordan coefficients for $L=0,1,2$ and $M=-L,-L+1, \ldots L$, define the transformation properties of (2), (3) and (44) under rotations. We also introduce the following notations $u_{m}^{\dagger}(\alpha=1 / 2)=p_{m}^{\dagger}$ and $u_{m}^{\dagger}(\alpha=-1 / 2)=n_{m}^{\dagger}$. In terms of the $p$ - and $n$-boson operators, the Weyl generators of the ladder representation of $U(3,3)$ are 9]

$$
p_{k}^{\dagger} p_{m}, \quad p_{k}^{\dagger} n_{m}^{\dagger}, \quad-n_{k} p_{m}, \quad-n_{m}^{\dagger} n_{k},
$$

which are obviously a subset of symplectic generators (2) - (4). The first-order Casimir operator of $U(3,3)$ is

$$
C_{1}[U(3,3)]=\sum_{k}\left(p_{k}^{\dagger} p_{k}-n_{k}^{\dagger} n_{k}\right)
$$

and does not differ essentially from the operator $T_{0}$ defined in 12$]$ :

$$
T_{0}=\frac{1}{2} C_{1}[U(3,3)]+\frac{3}{2} .
$$

The $U(3,3)$ irreps (ladder irreducible representations) contained in either (even) $<(1 / 2)^{6}>$ or $($ odd $)<(1 / 2)^{5} 3 / 2>$ irrep of $S p(12, R)$ can be denoted by the shorthand notation $[\nu], \nu \in Z$, defined as follows [9]:

$$
\begin{aligned}
{[\nu] } & =\left\{(1 / 2)^{2}, \nu+\frac{1}{2} ;(1 / 2)^{3}\right\} & \text { if } \nu>0 \\
& =\left\{(1 / 2)^{3} ;(1 / 2)^{2},-\nu+\frac{1}{2}\right\} & \text { if } \nu<0 \\
& =\left\{(1 / 2)^{3} ;(1 / 2)^{3}\right\}, & \text { if } \nu=0
\end{aligned}
$$


The branching rules can be written as

$$
<(1 / 2)^{6}>\downarrow \sum_{\nu=-\infty, \nu=\text { even }}^{+\infty} \oplus[\nu]
$$

and

$$
<(1 / 2)^{5} 3 / 2>\downarrow \sum_{\nu=-\infty, \nu=o d d}^{+\infty} \oplus[\nu] .
$$

In the present application we consider only the even irreducible representation of $S p(12, R)$.

The direct product $U_{p}(3) \otimes \overline{U_{n}(3)}$ subalgebra is defined by the subset of the number preserving generators (5) of $U(3,3)$, namely

$$
p_{k}^{\dagger} p_{m}, \quad-n_{m}^{\dagger} n_{k}
$$

Then, the combined (particle-hole) algebra $U^{*}(3)$ is simply expressed by the linear combination operators $A_{k m} \equiv$ $\left\{p_{k}^{\dagger} p_{m}-n_{m}^{\dagger} n_{k}\right\}$ of (13), which can also be defined in the following way 9 ]

$$
\begin{aligned}
& M=N_{p}-N_{n}, \\
& L_{M}=L_{M}^{p}+L_{M}^{n}, \\
& Q_{M}=Q_{M}^{p}-Q_{M}^{n} .
\end{aligned}
$$

The second order Casimir operator of $U^{*}(3)$ can be defined by

$$
C_{2}\left[U^{*}(3)\right]=\sum_{i j} A_{i j} A_{j i}
$$

The $S U^{*}(3)$ algebra is obtained by excluding the operator (14) which is the single generator of the $O(2)$ algebra, whereas the angular momentum algebra $S O(3)$ is generated by the generators $L_{M}$ only.

We can label the basis states according to the chain (11) as:

$$
\left|\nu ; N_{p}, N_{n} ;(\lambda, \mu) ; K L\right\rangle,
$$

where $\nu$ is the eigenvalue of the $U(3,3)$ first order Casimir operator, $N_{p}$ and $N_{n}$ label the $U_{p}(3) \otimes \overline{U_{n}(3)}$ irreps,$(\lambda, \mu)$ are the $S U^{*}(3)$ quantum numbers, $K$ is the multiplicity index in the reduction $S U(3) \supset S O(3)$, and $L$ is the angular momentum of the corresponding collective state.

The basis states associated with the even irreducible representation of the $S p(12, R)$ can be constructed by the application of powers of raising generators $F_{M}^{L}(\alpha, \beta)$ of the same group on the boson vacuum state. Each raising operator will increase the number of bosons $N$ by two. The $S p(12, R)$ classification scheme for the $S U^{*}(3)$ boson representations is shown on Table 1 The ladder representations of the non-compact algebra $U(3,3)$ act along the columns ("ladders") in the space of the boson representation of the $S p(12, R)$ algebra, defined through the eigenvalues $[\nu]$ of the first Casimir operator (6) of the $U(3,3)$ algebra. There exists a connection between this ladder rep-

\begin{tabular}{|c|c|c|c|c|c|c|c|}
\hline $\mid N \backslash \nu$ & \begin{tabular}{l|l}
$\cdots$ & 6 \\
\end{tabular} & 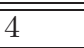 & $\overline{2}$ & $\overline{0}$ & $\overline{-1-2}$ & -4 & $\overline{-1-6}$ \\
\hline 0 & & & & $\overline{(0,0)}$ & & & \\
\hline 2 & & & $(2,0)$ & $\begin{array}{l}(1,1) \\
(0,0)\end{array}$ & $(0,2)$ & & \\
\hline 4 & & $(4,0)$ & $\begin{array}{l}(3,1) \\
(2,0)\end{array}$ & $\begin{array}{l}(2,2) \\
(1,1) \\
(0,0)\end{array}$ & $\begin{array}{l}(1,3) \\
(0,2)\end{array}$ & $(0,4)$ & \\
\hline 6 & $(6,0)$ & $\begin{array}{l}(5,1) \\
(4,0)\end{array}$ & $\begin{array}{l}(4,2) \\
(3,1) \\
(2,0)\end{array}$ & $\begin{array}{l}(3,3) \\
(2,2) \\
(1,1) \\
(0,0)\end{array}$ & $\begin{array}{l}(2,4) \\
(1,3) \\
(0,2)\end{array}$ & $\begin{array}{l}(1,5) \\
(0,4)\end{array}$ & $(0,6)$ \\
\hline & & & $\vdots$ & $\vdots$ & $\vdots$ & $\vdots$ & $\vdots$ \\
\hline
\end{tabular}
resentation ("vertical classification") and the boson representation of $U(6) \subset S p(12, R)$ ("horizontal classification"). Each row (fixed $N$ ) of the table corresponds to a given irreducible representation of the $U(6)$. Note that the number of bosons $N=N_{p}+N_{n}$ is not a good quantum number along the ladder representations of $U(3,3)$.
Table 1. Symplectic classification of the $S U^{*}(3)$ basis states.

\section{The energy spectrum}

The most general Hamiltonian with $S U^{*}(3)$ symmetry consists of the Casimir invariants of $S U^{*}(3)$ and its subgroup $S O(3)$

$$
H=a C_{2}\left[S U^{*}(3)\right]+b C_{2}[S O(3)],
$$

where

$$
C_{2}\left[S U^{*}(3)\right]=\frac{1}{6} Q^{2}+\frac{1}{2} L^{2}
$$

and the quadrupole operator $\mathrm{Q}$ is given by (16).

The spectrum of this Hamiltonian is determined by

$$
E=a\left(\lambda^{2}+\mu^{2}+\lambda \mu+3 \lambda+3 \mu\right)+b L(L+1) .
$$

We point out that there are very large degeneracies in the resulting energy spectrum caused by the large values of $\lambda$ and $\mu$, which is not observed in the real nuclear spectra.

In the present application we consider $S p(12, R)$ to be the group of the dynamical symmetry of the model. We make use of the following Hamiltonian:

$$
\begin{aligned}
H_{U(3,3)}= & a_{1} M^{2}+b\left(N_{n}^{2}-N_{p}^{2}\right) \\
& +a_{3} C_{2}\left[S U^{*}(3)\right]+b_{3} C_{2}[S O(3)],
\end{aligned}
$$

expressed as a linear combination of the Casimir operators of the different subgroups in the chain (1). The Hamiltonian (22) is diagonal in the basis (18). Then its eigenvalues that yield the spectrum of the nuclear systems are:

$$
\begin{aligned}
& E\left(\nu ; N_{p}, N_{n} ;(\lambda, \mu) ; L\right)=a_{1} \nu^{2}+b\left(N_{n}^{2}-N_{p}^{2}\right) \\
& +a_{3}\left(\lambda^{2}+\mu^{2}+\lambda \mu+3 \lambda+3 \mu\right)+b_{3} L(L+1) .
\end{aligned}
$$

The energy spectrum determined by Eq. 23. will be the starting point for our further calculations.

\section{Triaxial shapes in the IVBM}

In Ref. 9 it has been shown that the addition of different types of perturbations to the $S U^{*}(3)$ energy surface, in particular the addition of a Majorana interaction and an $O(6)$ term to the $S U^{*}(3)$ model Hamiltonian, produces a 
stable triaxial minimum in the potential energy surfaces. In present work we consider only the inclusion of a Majorana interaction to the model Hamiltonian and study the influence of the latter on the produced low-lying energy spectra. We expect that many experimental properties of some deformed even-even nuclei, exhibiting axially asymmetric features, to be explained with the perturbed Hamiltonian under consideration.

We present a schematic calculations starting with the Hamiltonian $\mathrm{H}$ (22) to which a perturbation term is added. The Hamiltonian which contains Majorana interaction is written in the form

$$
H=H_{U(3,3)}+a M_{3},
$$

where the Majorana operator is defined as

$$
M_{3}=2\left(p^{\dagger} \times n^{\dagger}\right)^{(1)} \cdot(p \times n)^{(1)}
$$

and it is related to the $U(3)$ second order Casimir invariant $C_{2}[U(3)]$, defined in Ref.[12], via the relation

$$
C_{2}[U(3)]=N(N+2)-2 M_{3} .
$$

The Hamiltonian $\mathrm{H}$ contains the pure $S U^{*}(3)$ symmetry, when only $a_{3} \neq 0$ in Eq.(22). The Majorana interaction can be written in the form

$$
\begin{aligned}
M_{3} & =\frac{2}{3}\left(N_{p}^{2}+N_{n}^{2}\right)+N_{p}+N_{n}-\frac{1}{3} M^{2}-\frac{1}{2} C_{2}\left[S U^{*}(3)\right] \\
& -\frac{1}{3}\left(\frac{15}{64}\left(C_{2}\left[S U^{*}(3)\right]-C_{2}\left[S U_{p}(3)\right]-C_{2}\left[\overline{S U_{n}(3)}\right]\right)\right. \\
& \left.-\frac{3}{32} \sqrt{30}\left[A^{(1,1)}(p, p) \times A^{(1,1)}(n, n)\right]^{(2,2)}\right) .
\end{aligned}
$$

It is evident that the only non-diagonal component of the Majorana interaction along the chain (10) is represented by the last term. It mixes different $S U(3)$ irreps.

In our application, the most important point is the identification of the experimentally observed states with a certain subset of basis states from symplectic extension of the model (Table 1). As in our previous applications of the symplectic IVBM, we use the algebraic concept of "yrast" states, introduced in 13. According to this concept we consider as yrast states the states with given $L$ that minimize the energy with respect to the number of vector bosons $N$ that build them. Since, the GSB in the triaxial nuclei is supposed to belong to the $S U^{*}(3)$ irreps of the type $\left(\lambda=N_{p}, \mu=N_{n}\right)$, we map the states of the GSB onto the ladder representation of $U(3,3)$ with $\nu=0$ (middle column of Table 1). The presented mapping of the experimental states onto the $S U(3)$ basis states, using the algebraic notion of yrast states, is a particular case of the so called "stretched" states [14, which in our case are defined as the states of the type $(\lambda, \mu)=\left(\lambda_{0}+k, \mu_{0}+k\right)$, where $k=0,2,4, \ldots$ In the symplectic extension of the IVBM the change of the number $k$, which is related in the applications to the angular momentum $L$ of the states, gives rise to the collective bands. Thus, explicitely the states of the GSB are identified with the $S U^{*}(3)$ multiplets $(\lambda, \mu)=(k, k)$, where $k=L$. The same type of stretched states $\left(\lambda_{0}+k, \mu_{0}+k\right)$ are associated with the states from the $\gamma$ band, where the symplectic band head structure of the considered band is determined by the initial number of phonons $N_{0}=\lambda_{0}+\mu_{0}=6\left(\lambda_{0}=2, \mu_{0}=4\right)$. Additionally, for the $\gamma$ band to each single $S U^{*}(3)$ irrep $(\lambda, \mu)$ ( $k$-fixed $)$ we put into correspondence two consecutive states with angular momentum $L$ and $L+1$, respectively. This choice allows us to reproduce the doublet structure of the $\gamma$-band. We note that the present choice of the $S U^{*}(3)$ multiplets associated with the states of the $\gamma$-band is quite similar to the phonon multiplet structure of the $\gamma$-band states within the framework of the IBM-1 in its $O(6)$ limit, where the states cluster in doublets differing in the $O(5)$ label $\tau$, which corresponds to the phonon-like quantum number $\Lambda$ in the $\gamma$-unstable model of Wilets and Jean [1].
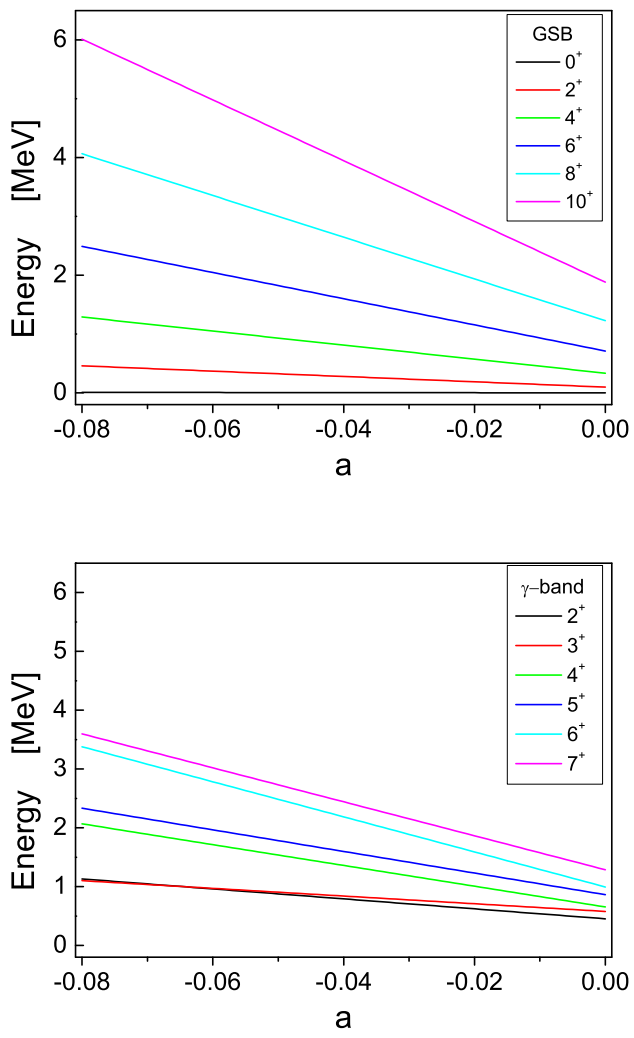

Fig. 1. (Color online) Energies of the ground and $\gamma$ bands as a function of the strength parameter $a$. The values of the rest model parameters are $a_{1}=0.10343 \mathrm{MeV}, b=-0.00274 \mathrm{MeV}$, $a_{3}=-0.00116 \mathrm{MeV}$ and $b_{3}=0.02092 \mathrm{MeV}$.

To show the influence of the Majorana interaction on the energy spectrum, we present the model calculations with the IVBM Hamiltonian (24) in which the Majorana term is included and diagonalyzed numerically. The evolution of the ground and $\gamma$ bands as a function of the strength parameter $a$ is shown in Fig. 1. From the figure one can see that the inclusion of the Majorana term does 
not change the level spacings of the ground state band and hence preserves its character. It can be also seen that the $\gamma$-rigid-like doublet structure of the $\gamma$-band is conserved for a wide interval of negative values of the parameter $a$, but for $a=0 \mathrm{MeV}$ (no mixing of the $S U(3)$ irreps) one obtains the well known $\gamma$-unstable-like structure. For $a \simeq-0.005 \mathrm{MeV}$ we obtain an intermediate situation with more regular spacing of the energy levels.

\section{Numerical results}

\subsection{Energy spectra}

Our theoretical considerations are applied for the calculation of the excitation energies of the ground and $\gamma$ bands in ${ }^{192} \mathrm{Os},{ }^{190} \mathrm{Os}$, and ${ }^{112} \mathrm{Ru}$, which are assumed in the literature to possess triaxial shapes. We determine the values of the model parameters $a_{1}, b, \alpha_{3}, \beta_{3}$, and $a$ by fitting the energies of the ground and $\gamma$ bands for the corresponding isotopes to the experimental data [15, using a $\chi^{2}$ procedure. The theoretical predictions, compared with experiment, are presented in Figures 2

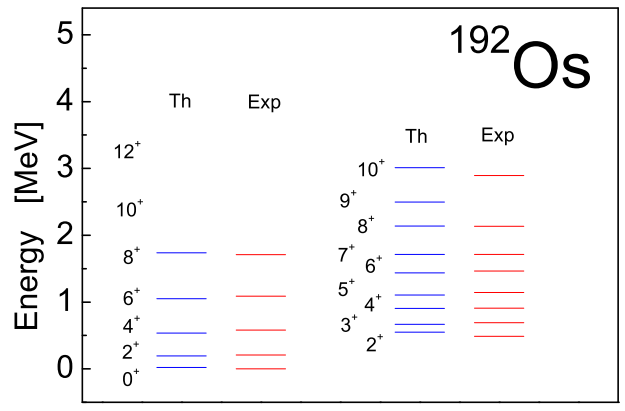

Fig. 2. (Color online) Excitation energies for GSB and $\gamma$ band of ${ }^{192} \mathrm{Os}$. The values of the rest model parameters are $a_{1}=$ $0.1034 \mathrm{MeV}, b=-0.0027 \mathrm{MeV}, a_{3}=-0.0011 \mathrm{MeV}, b_{3}=$ $0.0209 \mathrm{MeV}$, and $a=-0.0150 \mathrm{MeV}$.

There is a long-standing debate about the nature of the spectra of $O s$ isotopes. Some groups consider these nuclei as being $O(6)$-like with $\gamma$-unstable or $\gamma$-soft energy surfaces with a prolate minimum [16, 17, while other as asymmetric rotor [4, [18, [19, 20, which assumes rigidity in the $\gamma$ degrees of freedom. The Os isotopes considered here have been treated in terms of the IBM in the transition region from the rotor to the $\gamma$-unstable limit 21. In Ref. 22, these isotopes are considered as a textbook example of this transition. In Ref. 23 it was shown that the empirical deviations from the $O(6)$ limit of the IBM, in the Os-Pt region, can be interpreted by introducing explicitly triaxial degrees of freedom, suggesting a more complex and possibly intermediate situation between $\gamma$-rigid and $\gamma$-unstable properties. Indeed, as it

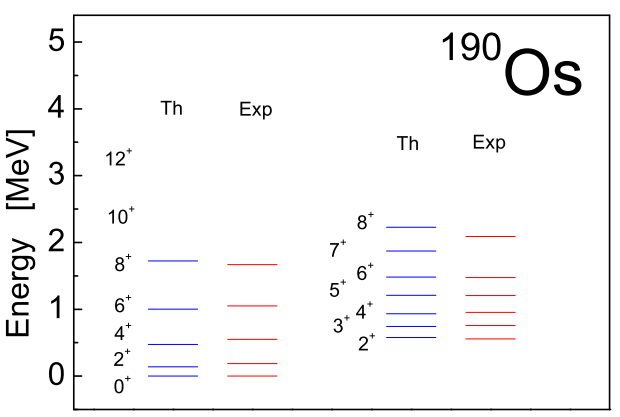

Fig. 3. (Color online) Excitation energies for GSB and $\gamma$ band of ${ }^{190} \mathrm{Os}$. The values of the rest model parameters are $a_{1}=$ $0.1127 \mathrm{MeV}, b=0.0004 \mathrm{MeV}, a_{3}=-0.0002 \mathrm{MeV}, b_{3}=0.0251$ $\mathrm{MeV}$, and $a=0 \mathrm{MeV}$.

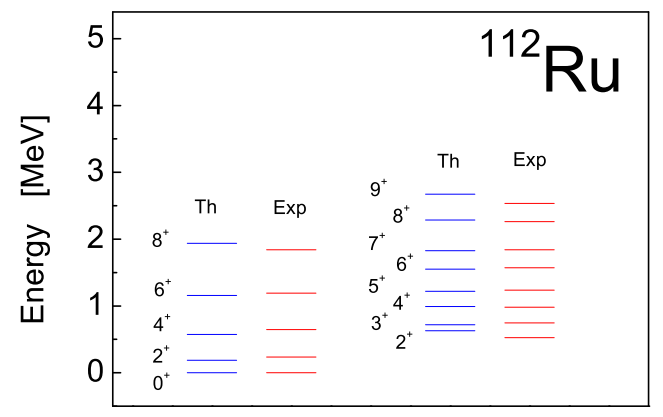

Fig. 4. (Color online) Excitation energies for GSB and $\gamma$ band of ${ }^{112} R u$. The values of the rest model parameters are $a_{1}=$ $0.1144 \mathrm{MeV}, b=-0.0056 \mathrm{MeV}, a_{3}=-0.0014 \mathrm{MeV}, b_{3}=$ $0.0212 \mathrm{MeV}$, and $a=-0.0209 \mathrm{MeV}$.

can be seen from the presented examples, the experimentally observed level spacings in the $\gamma$ band are more regular. In terms of the potentials, this means that the true potentials are $\gamma$-dependent.

Recently, a number of theoretical calculations [24, 25], [26, 27, 28 predict a tiny region of triaxiality between the prolate and oblate shapes in this mass region. The self-consistent Hartree-Fock-Bogoliubov calculations [26] with Gogny D1S and Skyrme SLy4 forces predict that the prolate to oblate transition takes place at neutron number $N=116\left({ }^{192} \mathrm{Os}\right)$.

The ${ }^{96-108} R u$ isotopes have also been described within the framework of IBM-1 as transitional between $U(5)$ and $O(6)$ limits 29], whereas in the Generalized Collective Model these nuclei are described as transitional between spherical and triaxial with a prolate onset for ${ }^{96} R u[30$. The collective structure of ${ }^{104-112} R u$ isotopes was also studied within the framework of the RTRM [31, 32. In the ${ }^{108,110,112} R u$, the experimental E2 branching ratios were found to be in overall agreement with those predicted 
by the RTRM calculations, but the calculated odd-even staggering in the $\gamma$ band shows disagreement with experiment. It has been recognized that in $\mathrm{Ru}$ nuclei the $\gamma$ dependence of the potential is intermediate between the two limiting cases of a $\gamma$-unstable and a $\gamma$-rigid rotor [31, 32.

From the Figs. 2-4 one sees that the calculated energy levels of both ground state and $\gamma$ bands agree rather well with the observed data and the doublet structure of the $\gamma$ band, although slightly pronounced in experiment, is correctly reproduced.

\subsection{The energy staggering}

A number of signatures of $\gamma$-unstable and $\gamma$-rigid structures in nuclei has been discussed [1, 2, [5]-8]. Many authors investigated the transition from the $\gamma$-unstable regime to a triaxial behavior. The two nuclear phases, as was mentioned, are characterized by different doublet structures in the $\gamma$ band. A useful quantity that distinguishes these two cases is the energy staggering signature [1, 2]:

$$
S(L)=\frac{[E(L)-E(L-1)]-[E(L-1)-E(L-2)]}{E\left(2_{g}^{+}\right)},
$$

where $E(L)$ stands for the energy of the state $L^{+}$belonging to the $\gamma$ band. The quantity $S(L)$ measures the displacement of the $(L-1)^{+}$level relative to the average of its neighbors, $L^{+}$and $(L-2)^{+}$, normalized to the energy of the first excited state of the ground band, $2_{g}^{+}$. The doublet structure is reflected in the sawtooth shape of the function $S(L)$.

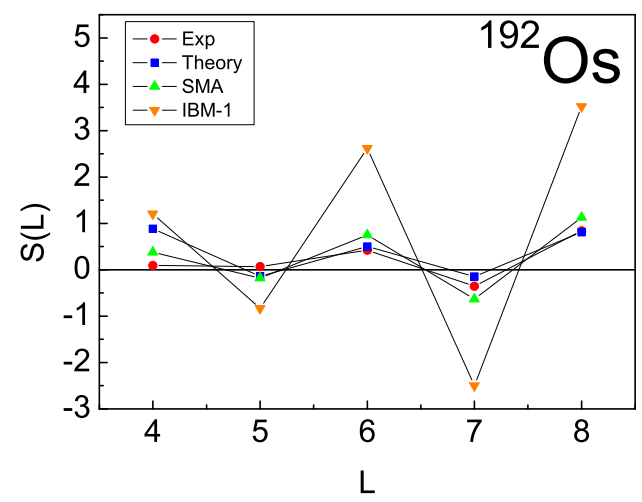

Fig. 5. (Color online) Calculated and experimental staggering of the $\gamma$ band in ${ }^{192}$ Os. The predictions of the sextic and Mathieu approach (SMA) 36] and the IBM-1 with a term quadratic in $(Q \otimes Q \otimes Q)_{0}$ 35. (IBM-1) are also shown.

Analysis of the experimental staggering in different isotopic chains reveals several different patterns [2] that can be categorized based on the standard limits discussed in the IBM. Just to mention few cases, the $X e, B a$ and $C e$ nuclei are well-known examples [23, 33, 33] of the transition between vibrational and $\gamma$-unstable structures that

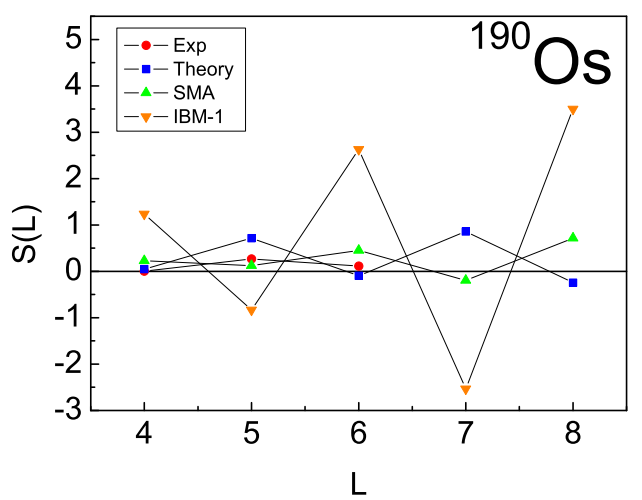

Fig. 6. (Color online) Calculated and experimental staggering of the $\gamma$ band in ${ }^{190} \mathrm{Os}$. The predictions of the sextic and Mathieu approach (SMA) 36 and the IBM-1 with a term quadratic in $(Q \otimes Q \otimes Q)_{0}$ 35] (IBM-1) are also shown.

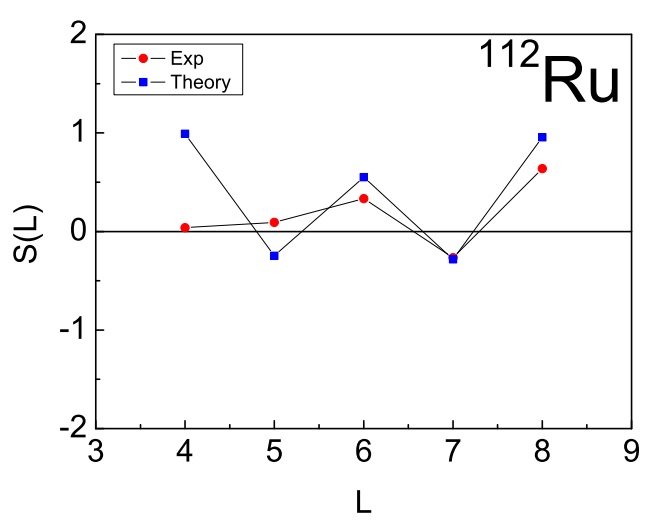

Fig. 7. (Color online) Calculated and experimental staggering of the $\gamma$ band in ${ }^{112} R u$.

show strong staggering with negative $S(L)$ values at even$L$ and positive $S(L)$ values at odd- $L$ spins. The heavy rareearth nuclei $(N>82)$, known to display an axially symmetric behavior, show a similar staggering pattern with a smaller overall magnitude than that observed in the $X e$, $B a$ and $C e$ isotopes. Nuclei that display staggering patterns very different from those described above are scarce and include, for example, ${ }^{192} \mathrm{Os}$ and ${ }^{112} \mathrm{Ru}$. These nuclei develop a staggering pattern where $S(L)$ is positive for even- $L$ and negative for odd- $L$ values, i.e. with the opposite phasing than in the other two cases mentioned above.

As shown in Ref. 2 the geometrical models and the IBM-based models can describe the basic trends observed in the experimental staggering. It is shown that the geometrical models that incorporate rigid triaxiality are characterized by strong staggering with positive values for even- $L$ and negative values for odd- $L$ spins. The staggering is largest for the RTRM where it increases linearly with $L$ and smallest for the models that use a harmonicoscillator $\beta^{2}$ potential. Similarly, the IBM shows a jump over to the triaxial region along the transition from $U(5)$ to $S U(3)$, characterized by the same staggering pattern 
as the one found in the geometrical models but with a smaller overall magnitude.

To see whether this signature is captured by the present approach, we plotted the function $S(L)$ within the framework of the IVBM for the nuclei under consideration in Figures 5-7 compared with the experimental data. For ${ }^{192} \mathrm{Os}$ and ${ }^{190} \mathrm{Os}$, the predictions of the IBM-1 with a term quadratic in $(Q \otimes Q \otimes Q)_{0} 35$ and sextic and Mathieu approach (SMA) 36] that incorporate $\gamma$-rigid structures are also shown. As can be seen from the figures, the present approach predicts a staggering pattern for ${ }^{192} \mathrm{Os}$ and ${ }^{112} R u$ similar to the one observed in the geometrical models and the IBM that incorporate triaxiality, and $\gamma$-unstable type for ${ }^{190} \mathrm{Os}$, respectively. For all nuclei under considerations, the phases of the observed staggering patterns are also correctly reproduced (in contrast to the SMA and IBM-1 in the case of ${ }^{190} O s$ ). For the two nuclei ${ }^{192} \mathrm{Os}$ and ${ }^{112} \mathrm{Ru}$, the $\gamma$-rigid staggering is well developed in the region $L \geq 5$ where also its magnitude increase with the spin. The latter suggests that the triaxiality evolves together with the collectivity.

\subsection{Energy surfaces}

The geometry associated with a given Hamiltonian can be obtained by the coherent state method. The standard approach to obtain the geometrical properties of the system is to express the collective variables in the intrinsic (body-fixed) frame of reference. Then the ground-state energy surface is obtained by calculating the expectation value of the boson Hamiltonian (24) with respect to the corresponding coherent states. In the case of IVBM, the (scaled) energy surfaces $\varepsilon(\rho, \theta)$ depend on two coherent state parameters $\rho$ and $\theta$, determining the "shape" of the nucleus 9, 12. The latter are related to the standard collective model "shape" parameters $\beta$ and $\gamma$. For more details we refer the reader to the Refs. 9], 12 .

We plot the ground state energy surfaces in ${ }^{192} \mathrm{Os}$, ${ }^{190} \mathrm{Os}$, and ${ }^{112} \mathrm{Ru}$ with the model parameters obtained in the fitting procedure in the form of contour plots in Fig. 8. From the figure one sees nearly $\gamma$-flat potentials with very shallow triaxial minima for the ground state in ${ }^{192} \mathrm{Os}$ and ${ }^{112} \mathrm{Ru}$, while in ${ }^{190} \mathrm{Os}$ a typical for the $O(6)$ limit $\theta$-unstable (or in IBM terms a $\gamma$-unstable) potential is observed, as should be for nuclei that show strong staggering with negative $S(L)$ values at even- $L$ and positive $S(L)$ values at odd- $L$ spins (see Fig 6 ). The triaxial minima are obtained at $\rho_{0} \approx 1$ and $\theta_{0}=90^{\circ}$ which corresponds to $\gamma_{\text {eff }}=30^{\circ}$. 9 . In other words, the potentials obtained in the present approach for ${ }^{192} \mathrm{Os}$ and ${ }^{112} \mathrm{Ru}$ are indeed $\gamma$-dependent, representing the case of mixing of $\gamma$ flat and $\gamma$-rigid structures.

Shallow triaxial minima in the energy surfaces of ${ }^{190} \mathrm{Os}$ and ${ }^{192} \mathrm{Os}$ are predicted in recent HFB calculations with Gogny D1S and Skyrme SLy4 forces [26], but with an $O(6)$-like doublet structure of the $\gamma$ bands. The IBM-2 energy surfaces [16, 17] derived from HFB ones with Gogny D1S forces predict a $\gamma$-soft energy surface with a prolate minimum for ${ }^{190} \mathrm{Os}$ and $\gamma$-unstable one for ${ }^{192} \mathrm{Os}$. The HF
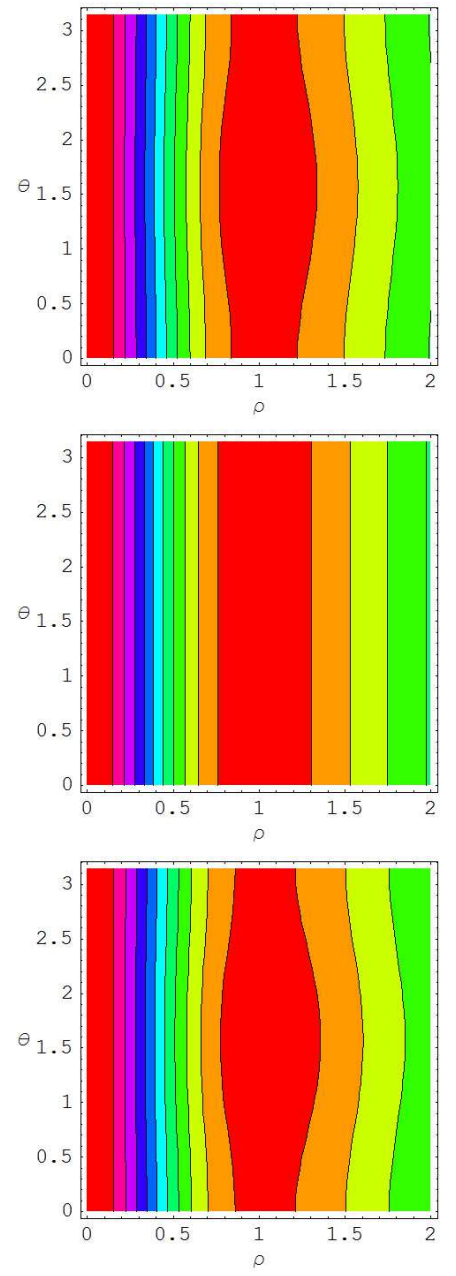

Fig. 8. (Color online) A contour plot of the scaled energy surfaces $\varepsilon(\rho, \theta)$ corresponding to the Hamiltonian (24) for ${ }^{192} \mathrm{Os}$, ${ }^{190} \mathrm{Os}$, and ${ }^{112} \mathrm{Ru}$, respectively. Only the region $\rho>0$ is depicted.

and IBM-2 calculations with $S k M^{*}$ forces 37] produce a triaxial minimum and $\gamma$-soft energy surface with a prolate minimum for ${ }^{112} R u$, respectively. A $\gamma$-soft energy surface with a prolate minimum is predicted also in 38. In 39, the Generalized Collective Model including up to sixth order (i.e. three-body) terms in $\alpha_{2 \mu}$ able to produce a stable triaxial minima predicts an energy surface with a triaxial minimum for ${ }^{112} R u$.

\section{Transition probabilities}

It is known that the comparison of the experimental data with the calculated transition probabilities is a more sensitive test for each model under consideration. In this respect, in this section we consider the electromagnetic properties of the IVBM with respect to the reduction chain (1).

The general approach required for the calculation of the matrix elements of the transition operators defined in respect to the chain (11), as well as its application to the more complicated and complex problem of reproducing 
the $B(E 2)$ transition probabilities between the collective states of the ground band in some isotopes exhibiting axially asymmetric features, is given in 40. Here, in addition to the intraband $E 2$ transitions between the states of the GSB we consider also the $E 2$ transitions between the states of the $\gamma$ band, as well as the interband $M 1$ transitions between the $\gamma$ and GSB in the two nuclei ${ }^{190} \mathrm{Os}$ and ${ }^{192} \mathrm{Os}$, for which there is enough available experimental data.

Transition probabilities are by definition $S O(3)$ reduced matrix elements of transition operators $T^{E 2}$ between the $|i\rangle$-initial and $|f\rangle$-final collective states

$$
B\left(E 2 ; L_{i} \rightarrow L_{f}\right)=\frac{1}{2 L_{i}+1}\left|\left\langle f\left\|T^{E 2}\right\| i\right\rangle\right|^{2}
$$

Using the tensorial properties of the $S p(12, R)$ generators and the mapping considered above, it is easy to define the $E 2$ transition operator between the states of the GSB and $\gamma$ band as 40:

$$
\begin{aligned}
T^{E 2}= & e\left[A_{[210]_{3}}^{\prime 20}+\theta\left(\left[\begin{array}{lc}
F \times]_{[2]_{3}[2]_{3}^{*}} & 20 \\
{[420]_{3}}
\end{array}\right.\right.\right. \\
& \left.\left.+[G \times G]_{[2]_{3}^{*}[2]_{3}} \quad \begin{array}{cc}
20 \\
{[420]_{3}}
\end{array}\right)\right],
\end{aligned}
$$

where the first tensor operator is the $S U^{*}(3)$ quadrupole operator and actually changes only the angular momentum with $\Delta L=2$ within a given irrep $(\lambda, \mu)$.

The tensor product

$$
\begin{aligned}
& {[F \times F]_{[2]_{3}[2]_{3}^{*}} \quad{ }^{20}[420]_{3}} \\
& =\sum C_{[2]_{3},[2]_{3}^{*}}^{[420]_{3}} C_{2}^{(2,0)(0,2)(2,2)} \underset{2}{2} C_{20,20}^{20} \\
& \times F_{[2]_{3}[0]_{3}^{*}} \quad \begin{array}{ll}
20 \\
{[2]_{3}}
\end{array} F_{[0]_{3}[2]_{3}^{*}} \quad \begin{array}{l}
20 \\
{[-2]_{3}}
\end{array}
\end{aligned}
$$

of the rasing generators of $S p(12, R)$ changes the number of bosons by $\Delta N=4$ and $\Delta L=2$.

The application actually consists of fitting the two parameters $e$ and $\theta$ of the transition operator $T^{E 2}$ (30) to experiment for each isotope. The experimental data [15] for the $B(E 2)$ transition probabilities between the states of the GSB and $\gamma$ band are compared with the corresponding theoretical results of the symplectic IVBM in Figure 9. For comparison, the theoretical predictions of IBM-2 41] in its $S U^{*}(3)$ limit, RTRM [42, sextic and Mathieu approach (SMA) 36, and $\gamma$-unstable model of Wilets and Jean 3 are also shown. From the figure one can see that all models presented reproduce the general trend of the experimental data, but nevertheless with the increasing of spin the RTRM, SMA, and $\gamma$-unstable model overestimate the observed experimental data well described by the IVBM. Note different values obtained for the model parameters for the two cases -with $\left({ }^{192} \mathrm{Os}\right)$ and without $\left({ }^{190} \mathrm{Os}\right)$ mixing of the $S U(3)$ multiplets.

Next, we consider the interband $M 1$ transitions between the GSB and $\gamma$ band for which there is a very few
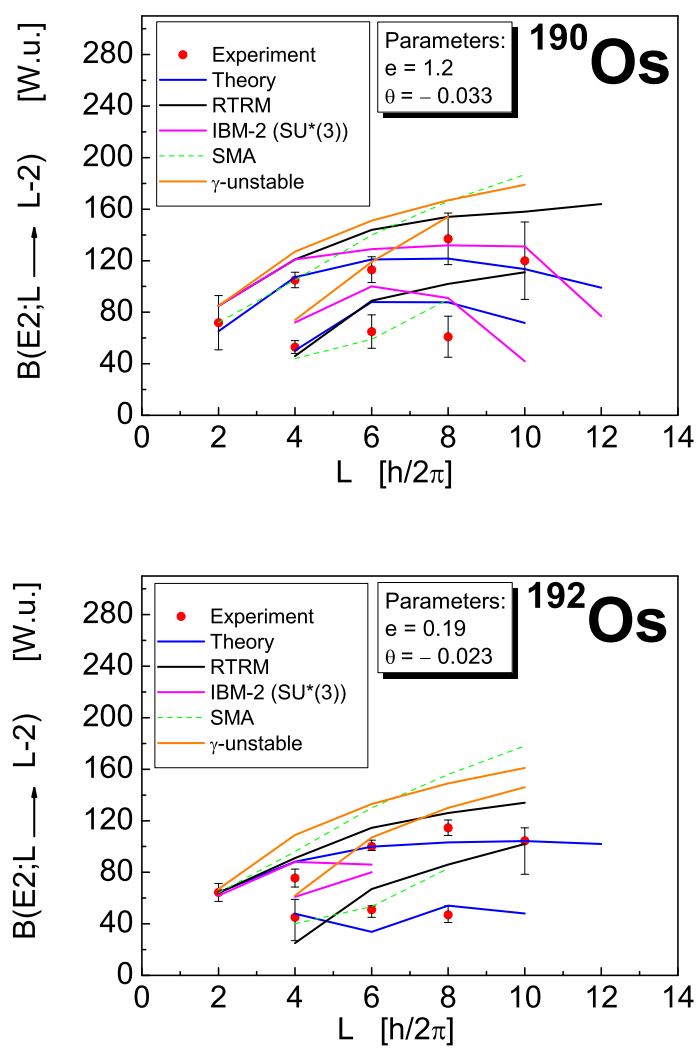

Fig. 9. (Color online) Comparison of theoretical and experimental values for the $B(E 2)$ transition probabilities between the collective states of the ground state band and $\gamma$ band in ${ }^{190} \mathrm{Os}$ and ${ }^{192} \mathrm{Os}$, respectively. The theoretical results of some other collective models which accommodate the $\gamma$-rigid or $\gamma$ unstable structures are also shown.

experimental data. Using the tensorial properties of the basis states and the indetification of the experimentally observed states of the GSB and $\gamma$ band with the subset of stretched states from the $\nu=0$ and $\nu=-2$ irreps of $U(3,3)$, respectively, we can define as an appropriate the following $M 1$ transition operator lying in the enveloping algebra of $S p(12, R)$ :

$$
T^{M 1}=g_{F G}\left([L \times F]_{[4,3,0]_{3}}^{10}+[L \times G]_{[4,1,0]_{3}}^{10}\right),
$$

where the operators $F, G$ and $L$ are defined by Eqs. (2), (3) and (15), respectively. (For more details concerning the tensor properties of the $S p(12, R)$ algebra generators and the construction of the symplectic basis see Ref.40.)

In Figure 10 we compare our theoretical predictions for the interband M1 transitions bitween the states of the GSB and $\gamma$ band with experiment [15] for the two nuclei ${ }^{190} \mathrm{Os}$ and ${ }^{192} \mathrm{Os}$, respectively. Despite the very poor experimental information, one sees a good reproduction of the $B(M 1)$ transition probabilities for the two nuclei under consideration.

The results obtained for the transition probabilities considered in this section also prove the correct mapping 

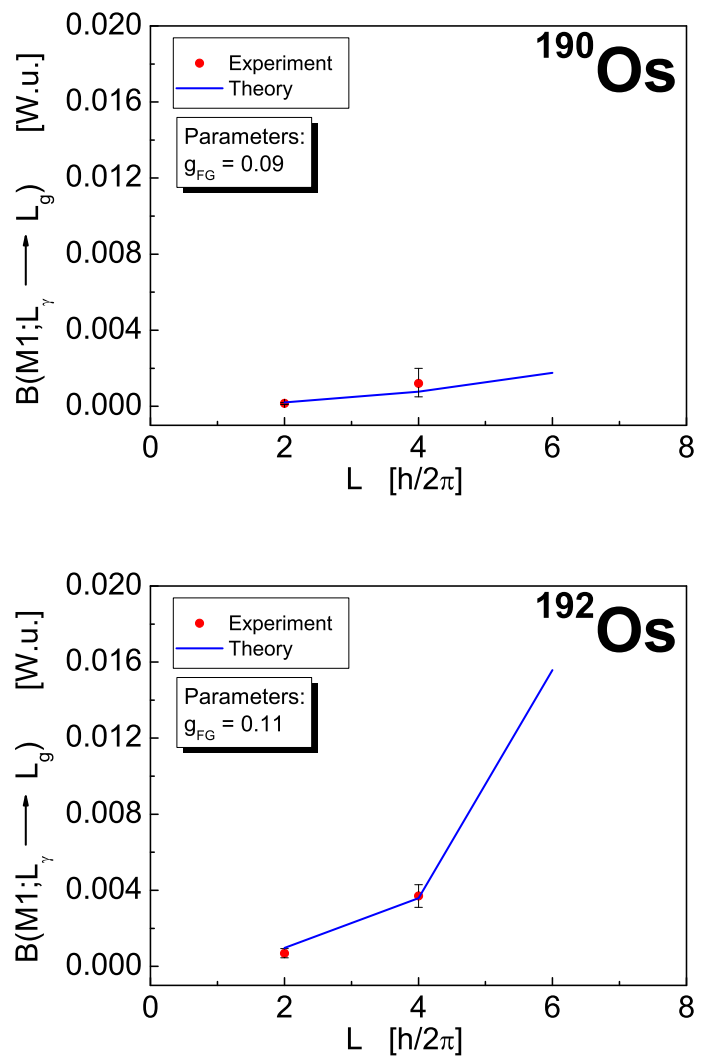

Fig. 10. (Color online) Comparison of theoretical and experimental values for the interband $B(M 1)$ transition probabilities between the ground state band and $\gamma$ band in ${ }^{190} \mathrm{Os}$ and ${ }^{192} \mathrm{Os}$, respectively.

of the basis states to the experimentally observed ones and reveal the relevance of the IVBM in the description of nuclei that exhibit axially asymmetric features in their spectra. Certainly, the used dynamical symmetry of the IVBM needs to be further explored by more detailed comparison of its predictions with the available experimental data.

\section{Summary}

In the present work, we apply one of the dynamical symmetry limits of the two-fluid Interacting Vector Boson Model, defined through the chain $S p(12, R) \supset U(3,3) \supset$ $U_{p}(3) \otimes \overline{U_{n}(3)} \supset S U^{*}(3) \supset S O(3)$, for the description of some even-even nuclei, possessing axial asymmetry. We have investigated the effect of the introduction of a Majorana interaction to the $S U^{*}(3)$ model Hamiltonian on the excitation energies of the ground and $\gamma$ bands. The latter introduces a potential which has a minimum at $\gamma=30^{\circ}$ and change the $\gamma$-band doublet structure from that of $\gamma$-unstable to that of $\gamma$-rigid type. This allows for the description of these two limiting cases, as well as the situation in between, which is characterized by more uni- form energy level spacings in the $\gamma$-band, and described actually by $\gamma$-dependent potentials.

The theoretical predictions are compared with the experimental data for ${ }^{192} \mathrm{Os},{ }^{190} \mathrm{Os}$, and ${ }^{112} \mathrm{Ru}$ isotopes. It is shown that by taking into account the full symplectic structures in the considered dynamical symmetry of the IVBM, the proper description of the energy spectra and the $\gamma$-band energy staggering of the nuclei under considerations can be achieved. The obtained results show that the potential energy surfaces for the following two nuclei ${ }^{192} \mathrm{Os}$ and ${ }^{112} \mathrm{Ru}$, possess almost $\gamma$-flat potentials with very shallow triaxial minima, suggesting a more complex and intermediate situation between $\gamma$-rigid and $\gamma$-unstable structures.

Symplectic dynamical symmetries provide for larger classification spaces and richer subalgebraic structures allowing to incorporate more complicated structural effects in nuclear spectra. Additionally, the algebraic structure of the model allows both the basis states and the transition operators to be defined as tensors in respect to the considered dynamical symmetry which simplifies the calculation of the transition probabilities by using a generalized version of the Wigner-Eckart theorem. This allows the model to be further tested on the more complicated problem of reproducing the transition probabilities between the collective states attributed to the basis states of the Hamiltonian. Exploiting this, the theory is further applied for the calculation of the absolute $B(E 2)$ intraband transition probabilities between the states of the ground state band and $\gamma$ band, as well as the $B(M 1)$ interband transition probabilities between the states of the GSB and $\gamma$ band for the two nuclei ${ }^{192} \mathrm{Os}$ and ${ }^{190} \mathrm{Os}$ for which there is enough available experimental data. The theoretical predictions are compared with experiment and for the $B(E 2)$ values with the predictions of some other collective models incorporating the $\gamma$-rigid or $\gamma$-unstable structures.

The results obtained for the energy levels, the energy staggering of the $\gamma$ band and the transition probabilities of the considered nuclei prove the correct mapping of the basis states to the experimentally observed ones and reveal the relevance of the used dynamical symmetry of IVBM in the description of nuclei exhibiting axially asymmetric features in their spectra.

\section{References}

1. N. V. Zamfir and R. F. Casten, Phys. Lett. B260, 265 (1991).

2. E. A. McCutchan, D. Bonatsos, N. V. Zamfir, and R. F. Casten, Phys. Rev. C76, 024306 (2007).

3. L. Wilets and M. Jean, Phys. Rev. 102, 788 (1956).

4. A. S. Davydov and G. F. Filippov, Nucl. Phys. 8, 237 (1958).

5. Liao Ji-zhi, Phys. Rev. C51, 141 (1995).

6. C.Bihari et al., Phys. Scr. 77, 055201 (2008).

7. C. Bihari et al., Phys. Scr. 78, 045201 (2008).

8. Mani Varshney, Phys. Scr. 83, 015201 (2011).

9. H. G. Ganev, Phys. Rev. C84, 054318 (2011).

10. V. Bargmann and M. Moshynsky, Nucl. Phys. 18, 697 (1960). 
11. V. Bargmann and M. Moshynsky, Nucl. Phys. 23, 177 (1961).

12. H. G. Ganev, Phys. Rev. C83, 034307 (2011).

13. H. Ganev, V. P. Garistov, and A. I. Georgieva, Phys. Rev. C 69, 014305 (2004)

14. D. J. Rowe, Rep. Prog. Phys. 48, 1419 (1985).

15. National Nuclear Data Center (NNDC), http://www.nndc.bnl.gov/

16. K. Nomura et al., Phys. Rev. C 83, 054303 (2011).

17. K. Nomura et al., Phys. Rev. C 84, 054316 (2011)

18. N. Redon et al., Phys. Lett. B181, 223 (1986).

19. W. Boeglin et al., Nucl. Phys. A 477, 289 (1988).

20. P. Sarriguren, R. Rodrguez-Guzman, L. M. Robledo, Phys. Rev. C 77, 064322 (2008).

21. R. F. Casten and J. A. Cizewski, Nucl. Phys. A 309, 477 (1978).

22. R. F. Casten, Nuclear Structure from a Simple Perspective (Oxford University, Oxford, 1990).

23. R.F. Casten, P. Von Brentano, K. Heyde, P. Van Isacker, J. Jolie, Nucl. Phys. A 439, 289 (1985).

24. P. Bonche et al., Nucl. Phys. A500, 308 (1989).

25. L. Fortunato, C. E. Alonso, J. M. Arias, J. E. GarciaRamos, and A. Vitturi, Phys. Rev. C 84, 014326 (2011).

26. L. M. Robledo, R. Rodriguez-Guzman, and P. Sarriguren, J. Phys. G: Nucl. Part. Phys. 36, 115104 (2009).

27. K. Nomura et al., Phys. Rev. C 83, 014309 (2011).

28. K. Nomura et al., Phys. Rev. C 84, 014302 (2011).

29. A. Frank, P. Van Isacker, and P. D. Warner, Phys. Lett. B 197, 474 (1987).

30. D. Troltenier et al., Z. Phys. A 338, 261 (1991).

31. J. Stachel et al, Z. Phys. A316, 105 (1984).

32. J. A. Shannon et al., Phys. Lett. B 336, 136 (1994).

33. G. Puddu, O. Scholten, T. Otsuka, Nucl. Phys. A 348, 109 (1980).

34. N.V. Zamfir, R.F. Casten, Phys. Lett. B 152, 22 (1985).

35. G. Thiamova, Eur. Phys. J. A 45, 81 (2010).

36. A. A. Raduta and P. Buganu, Phys. Rev. C 83, 034313 (2011).

37. K. Nomura et al., Phys. Rev. C 81, 044307 (2010).

38. J. Aysto et al., Nucl. Phys. A515, 365 (1990).

39. D. Troltenier et al., Nucl. Phys. A601, 56 (1996).

40. H. G. Ganev, Phys. Rev. C 86, 054311 (2012).

41. N. R. Walet and P. J. Brussaard, Nucl. Phys. A474, 61 (1987).

42. H. Toki and A. Faessler, Z. Phys. A 276, 35 (1976). 